\title{
Multiparticle Interfacial Drag in Equiaxed Solidification
}

\author{
C.Y. WANG, S. AHUJA, C. BECKERMANN, and H.C. de GROH III
}

A physical model is proposed for the solid/liquid interfacial drag in both globular and dendritic equiaxed solidification. By accounting for the presence of multiple particles and the nonsphericity and porosity of the individual equiaxed crystals, a drag correlation is developed, which is valid over the full range of solid volume fractions. It is shown that neither the solid liquid interfacial area concentration nor the grain size alone is adequate to characterize the interfacial drag for equiaxed dendritic crystals in both the free particle and packed bed regimes; thus, the present model is based on a multiple length scale approach. The model predictions are compared to previous analytical and numerical results as well as to experimental data available in the literature, and favorable agreement is achieved.

\section{INTRODUCTION}

RELATIVE motion between solid and liquid during alloy solidification is one of the most critical transport phenomena associated with macrosegregation and microstructural development in solidification of metal alloys. In order to predict the relative movement as well as its ensuing effects in a solidifying alloy, accurate knowledge of the solid/liquid interfacial drag over the entire range of solid volume fractions is required. ${ }^{[1]}$ Two regimes can be distinguished in solidifying alloy castings: (1) the free particle regime, where crystals can move in the melt, and (2) at higher solid fractions, the packed bed regime, where the crystals are stationary and the melt penetrates through the bed.

Numerous studies on solid/liquid interfacial drag have been carried out due to this drag's importance in many engineering disciplines, as discussed in Ahuja. ${ }^{[2]}$ Within the context of equiaxed alloy solidification, notable works include those by Piwonka and Flemings, ${ }^{[3]}$ Murakami and Okamoto, ${ }^{[4]}$ Poirier and Ganesan, ${ }^{[5]}$ and de Groh et al..$^{[6] *}$ Piwonka and Flemings ${ }^{[3]}$ were the first

\footnotetext{
*After submission of the present article, the authors became aware of two very recent articles by Poirier and co-workers on the permeability of equiaxed mushy zones. ${ }^{[28,29]}$
}

to measure the permeability in $\mathrm{Al}-4.5$ wt pct $\mathrm{Cu}$ alloys with equiaxed dendritic structures. Their data are unfortunately of limited use because the microstructures were not well characterized, so that a relationship between the permeability and the geometrical parameters of dendrite structures cannot be fully established. Murakami and Okamoto, ${ }^{[4]}$ using borneol-paraffin alloys, reported experimental data for the permeability of a mushy zone consisting of globular grains, and more recently, Poirier and Ganesan ${ }^{[5]}$ measured the permeability of $\mathrm{Al}-\mathrm{Cu}$ equiaxed systems with both globular

C.Y. WANG, Research Assistant, and C. BECKERMANN, Associate Professor, are with the Department of Mechanical Engineering, University of Iowa, Iowa City, IA 52242-1527. S. AHUJA, formerly Graduate Research Assistant, Department of Mechanical Engineering, University of Iowa, is Software Development Engineer, Engineering Mechanics Research Corporation, Troy, MI 48098. H.C. de GROH III, Materials Research Engineer, is with NASA Lewis Research Center, Cleveland, $\mathrm{OH} 44135$.

Manuscript submitted December 1, 1993. and dendritic-globular structures. These two recent studies are restricted to solid volume fractions higher than 0.5 (i.e., the packed bed regime). In the other extreme, Zakhem et al. ${ }^{[7]}$ determined the free settling characteristics of single equiaxed crystals and dendrite fragments and de Groh et al. ${ }^{[6]}$ proposed a correlation for the interfacial drag.

It is apparent from this literature survey that a general correlation capable of predicting the interfacial drag in an equiaxed system for all solid volume fractions is still lacking. Such a correlation, however, represents a key constitutive relationship for solidification models that include fluid flow in the mushy zone and the transport of free equiaxed crystals. The present article, therefore, is intended to develop a physical model for the calculation of the interfacial drag in a system composed of multiple equiaxed crystals. Both globular and dendritic morphologies are considered. Three important effects that dictate flows around and through dendrites in the system under consideration are addressed in the model: the presence of multiple crystals, the nonsphericity of the crystal envelopes, and the porosity of equiaxed dendritic crystals. Furthermore, because for equiaxed dendrites there is a potentially large disparity between the size of the solidliquid interfacial area and the overall grain size, the present model explicitly accounts for multiple length scales. Finally, the model is compared to previous analytical and semianalytical results and validated by the limited experimental data available in the literature.

\section{MODEL DEVELOPMENT}

\section{A. Basic Considerations}

This article is a continuation of the work by de Groh et al.$^{[6]}$ on free settling of single equiaxed crystals. The present model extends the previous work by accounting for the presence of multiple crystals. Use is made of the "porous dendrite envelope" concept introduced by de Groh et al ${ }^{[6]}$ or more generally, the multiphase approach proposed by Wang and Beckermann. ${ }^{[8]}$ The dendrite envelope is defined as a smooth surface connecting the primary and secondary arm tips and contains both the solid dendrite and the interdendritic liquid. Hence, a control element consisting of multiple equiaxed dendritic crystals shown in Figure 1(a) can be viewed to be occupied by three phases: solid (s), interdendritic liquid 


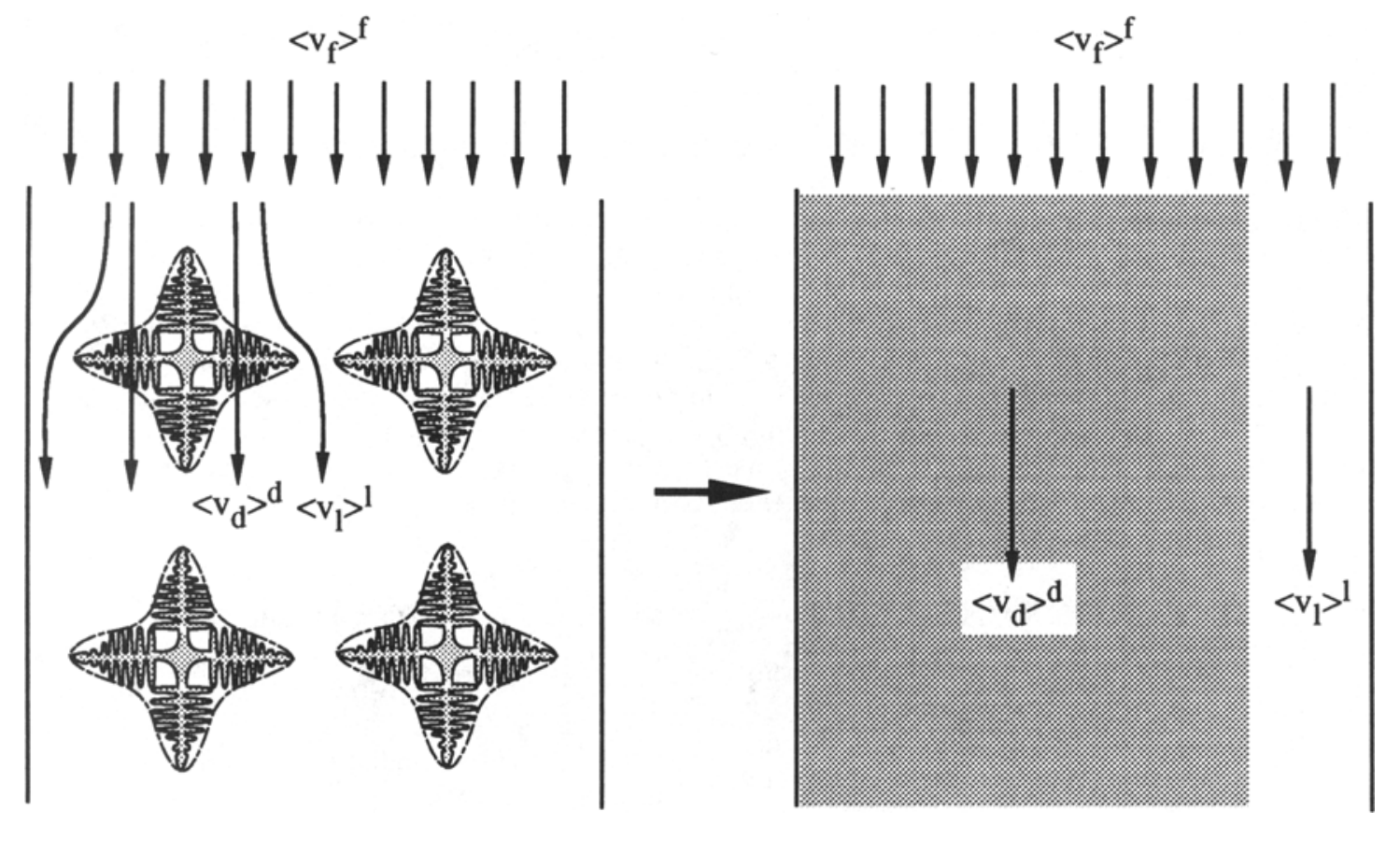

(a)

(b)

Fig. 1-Schematics of $(a)$ the physical problem: flow through a control element of equiaxed dendrites; and $(b)$ the model: flow through a porous/plain composite channel.

(d), and extradendritic liquid (l), with the two liquid phases separated by the dendrite envelope. The reader is urged to refer to References 8 through 10 for a more thorough discussion of the multiphase approach as well as its various applications. The liquid phases are associated with different interfacial length scales and possess different flow behaviors. The fluid flow around the dendrites is mainly controlled by the extradendritic liquid fraction and the nonspherical shape and size of the dendrite envelope, while the flow through the dendrites is determined by the permeability within the porous dendrite envelope. These two flows interactively affect the drag force experienced by the dendrites. The globular crystal represents a limiting case of an equiaxed dendritic crystal, in which the interdendritic liquid is absent and the envelope coincides with the solid/liquid interface.

With reference to Figure 1(a), several important parameters necessary for the following model development are introduced:

(1) The interfacial area concentrations, $S_{s}$ and $S_{e}$, denoting the surface areas of the solid/interdendritic liquid and dendrite envelope interfaces, respectively, divided by the total volume, $V_{0}$, of the control element shown in Figure 1(a). The inverses of $S_{s}$ and $S_{e}$ are the aforementioned two interfacial length scales that characterize dendritic solidification. For highly dendritic crystals, the difference between $S_{s}$ and $S_{e}$ can be several orders of magnitude. In globular solidification, $S_{s}$ and $S_{e}$ coincide. (2) The sphericity of the dendrite envelope, $\phi_{e}$, defined as the ratio of the surface area of a hypothetical sphere of the same volume as the dendrite envelope to the actual envelope surface area. The sphericity (lying between zero and unity) is a measure of the deviation of the dendrite envelope shape from a perfect sphere, with $\phi_{e}=1$ representing a spherical dendrite envelope.

(3) The volume fraction of phase $k, \varepsilon_{k}$, designating the fraction of the phase volume within the control element, where $k$ corresponds to $s$ (solid phase), $d$ (interdendritic liquid phase), or $l$ (extradendritic liquid phase). Hence, $\varepsilon_{s}+\varepsilon_{d}+\varepsilon_{l}=1$. For globular crystals, $\varepsilon_{d}=0$.

(4) The volume-averaged intrinsic velocity of phase $k$, $\left\langle v_{k}\right\rangle^{k}$. Physically, the term $\left\langle v_{k}\right\rangle^{k}$ is nothing else but the average velocity of a phase in the control element; e.g., $\left\langle v_{s}\right\rangle^{s}$ is the solid velocity.

In addition, the internal solid fraction, $\varepsilon_{s i}$, within the porous dendrite envelope, the total liquid fraction, and the total liquid velocity are defined, respectively, as

$$
\begin{aligned}
\varepsilon_{s i} & =\varepsilon_{s} /\left(\varepsilon_{s}+\varepsilon_{d}\right) \\
\varepsilon_{f} & =\varepsilon_{d}+\varepsilon_{l}
\end{aligned}
$$

and

$$
\rho_{f} \varepsilon_{f}\left\langle v_{f}\right\rangle^{f}=\rho_{d} \varepsilon_{d}\left\langle v_{d}\right)^{d}+\rho_{l} \varepsilon_{d}\left\langle v_{l}\right\rangle^{l}
$$

where subscript $f$ denotes the total liquid, consisting of the inter- and extradendritic liquids. In the following, it is further implicitly assumed that thermophysical properties of the inter- and extradendritic liquids are identical.

Traditionally, there have been different approaches to the modeling of the interfacial drag in a multiparticle system. For high solid fractions (i.e., the packed bed regime), the porous medium approach is often adopted, with the permeability representing a key parameter. ${ }^{[11,12]}$ 
While at low solid fractions (i.e., the free particle regime), the submerged object model is more frequently used. ${ }^{[6,13]}$ In the latter approach, a multiparticle system is viewed as an assemblage of submerged objects, and the interfacial drag is calculated by modifying the Stokes law for a single spherical particle. Recently, however, it has been shown that both approaches can be unified, thereby resulting in a generalized drag model (valid for all solid fractions) for solid sphere systems. ${ }^{[14]}$ Indeed, in the submerged object approach, the total drag force in a unit volume of the dendrite system shown in Figure 1(a) can be written as ${ }^{[2]}$

$$
M^{d}=\frac{18\left(1-\varepsilon_{l}\right) \mu_{f} C_{\varepsilon}}{d_{e}^{2}} \varepsilon_{f}^{2}\left(\left\langle v_{f}\right\rangle^{f}-\left\langle v_{s}\right\rangle^{s}\right)
$$

where the drag is based on the superficial relative velocity, namely, $\varepsilon_{f}\left(\left\langle v_{f}\right\rangle^{\gamma}-\left\langle v_{s}\right\rangle^{s}\right)$, and $d_{e}$ is the diameter of an equivalent sphere having the same volume as the dendrite envelope: ${ }^{: 8]}$

$$
d_{e}=\frac{6\left(1-\varepsilon_{l}\right)}{\phi S_{e}}
$$

The settling ratio of a particle in a multiparticle system, $C_{\varepsilon}$, accounts for all departures from the idealized single solid sphere settling as described by Stokes law. Under the low particle Reynolds number condition, these include (1) the porosity of and (2) the nonsphericity of the dendrite envelope as well as (3) the presence of other particles.

The drag force, $M^{d}$, must also be equal to the pressure gradient $\Delta p / \Delta x$, and the latter can be written as

$$
\frac{\Delta p}{\Delta x}=\frac{\mu_{f}}{K} \varepsilon_{f}\left(\left\langle v_{f}\right\rangle^{f}-\left\langle v_{s}\right\rangle^{s}\right)
$$

according to Darcy's law for flow in packed beds. Equating Eqs. [4] and [6] yields

$$
K=\frac{d_{e}^{2}}{18\left(1-\varepsilon_{l}\right) C_{\varepsilon}}
$$

This relation demonstrates a mathematical equivalence between the overall permeability, $K$, and the settling ratio, $C_{\varepsilon}$, and furthermore implies the equivalency of the porous medium and submerged object approaches. Note from Eqs. [4] and [7] that the knowledge of either $K$ or $C_{\varepsilon}$ is necessary to determine the interfacial drag.

\section{B. Overall Permeability}

To determine the overall permeability, $K$, it is assumed that the flow through the multidendrite system shown in Figure 1(a) is equivalent to the one through a composite channel that consists of a fine porous layer (modeling the interdendritic region) adjacent to a thin plain layer (modeling the extradendritic region). This is shown in Figure 1(b). The porous sublayer is characterized by an interdendritic permeability, $K_{d}$, which is a measure of the interdendritic flow rate under a certain pressure gradient. The extradendritic flow is mainly determined by the size of the extradendritic region, so that an extradendritic permeability, $K_{l}$, can be defined as the flow resistance of the composite channel when the interdendritic region is fully solid. Hence, the following two limiting cases exist:

$$
K=\left(1-\varepsilon_{l}\right) K_{d} \quad \text { when } K_{l}=0
$$

and

$$
K=K_{i} \quad \text { when } K_{d}=0
$$

The term appearing before $K_{d}$ in Eq. [8] is a volumetric factor to transform $K_{d}$ into $K$ when the extradendritic region is impermeable. According to the general correlation method proposed by Churchill and Usagi, ${ }^{115]}$ which has found many successful applications in a spectrum of engineering disciplines, a generalized correlation for $K$ can be constructed that is of the form

$$
K=\left\{\left[\left(1-\varepsilon_{l}\right) K_{d}\right]^{n}+K_{l}^{n}\right\}^{1 / n}
$$

where $n$ is an index to be determined. Equation [10] identically reduces to the limiting cases expressed by Eqs. [8] and [9]. To facilitate the following derivation, a dimensionless diameter is introduced as in Neale et al.:[16]

$$
\beta=\frac{d_{e}}{2 \sqrt{K}}=\frac{3\left(1-\varepsilon_{l}\right)}{\phi_{e} S_{e} \sqrt{K}}
$$

Then, the general correlation, Eq. [10], can be rewritten as

$$
\beta=\frac{\beta_{d}}{\left[\left(1-\varepsilon_{l}\right)^{n}+\left(\beta_{d} / \beta_{l}\right)^{2 n}\right]^{1 / 2 n}}
$$

where $\beta_{d}$ and $\beta_{l}$ are likewise defined by Eq. [11] with $K$ replaced by $K_{d}$ and $K_{l}$, respectively.

\section{Flow Partition Coefficient}

Another useful piece of information can now be obtained: the distribution of the liquid flow rate in the interand extradendritic regions. A flow partition coefficient, $\kappa_{v}$, is defined as the ratio of the liquid flow rate passing through the equiaxed dendrites to the total flow rate through the whole system, namely,

$$
\varepsilon_{d}\left(\left\langle v_{d}\right\rangle^{d}-\left\langle v_{s}\right\rangle^{s}\right)=\kappa_{v} \varepsilon_{f}\left(\left\langle v_{f}\right\rangle^{f}-\left\langle v_{s}\right\rangle^{s}\right)
$$

and

$$
\varepsilon_{l}\left(\left\langle v_{l}\right\rangle^{l}-\left\langle v_{s}\right\rangle^{s}\right)=\left(1-\kappa_{v}\right) \varepsilon_{f}\left(\left\langle v_{f}\right\rangle^{f}-\left\langle v_{s}\right\rangle^{s}\right)
$$

The coefficient $\kappa_{v}$ is also called the fluid collection efficiency of porous aggregates in chemical engineering. ${ }^{[17]}$ Assuming creeping flow in the overall composite system as well as in the interdendritic region under the low particle Reynolds number condition, the total liquid and the interdendritic liquid velocities can be linearly related to the common pressure gradient imposed, respectively, as

$$
\begin{gathered}
\left(1-\varepsilon_{s i}\right)\left(\left\langle v_{d}\right\rangle^{d}-\left\langle v_{s}\right\rangle^{s}\right)=\frac{K_{d}}{\mu_{d}}\left(\frac{\Delta p}{\Delta x}\right) \\
\varepsilon_{f}\left(\left\langle v_{f}\right\rangle^{f}-\left\langle v_{s}\right\rangle^{s}\right)=\frac{K_{f}}{\mu_{f}}\left(\frac{\Delta p}{\Delta x}\right)
\end{gathered}
$$


where the left-hand sides of Eqs. [15] and [16] represent the superficial relative velocities as required by Darcy's law. Substituting Eqs. [15] and [16] into [13] and further noting Eq. [11] then yields

$$
\kappa_{v}=\left(1-\varepsilon_{l}\right)\left(\beta / \beta_{d}\right)^{2}
$$

As a constitutive relation, Eq. [17] can greatly simplify the solution of the multiphase model recently proposed in Reference 18. This is because only the momentum equation for the total liquid phase needs to be solved, and the individual liquid velocity fields, $\left\langle v_{d}\right\rangle^{d}$ and $\left\langle v_{l}\right\rangle^{l}$ can be algebraically obtained from Eqs. [13] and [14] together with $\kappa_{v}$ calculated from Eq. [17].

Both the overall permeability $K$ (or $\beta$ ) and the flow partition coefficient $\kappa_{v}$ depend on three quantities: $\beta_{d}, \beta_{l}$, and $n$, whose evaluations follow.

\section{Evaluation of the Interdendritic Permeability}

The interdendritic permeability, $K_{d}$, within the dendrite envelope can be estimated from the KozenyCarman equation, ${ }^{[11]}$ namely,

$$
K_{d}=\frac{\left(1-\varepsilon_{s i}\right)^{3}}{5\left(A_{s} / V_{e}\right)^{2}}=\frac{\left(1-\varepsilon_{s i}\right)^{3}\left(1-\varepsilon_{l}\right)^{2}}{5 S_{s}^{2}}
$$

where $A_{s}$ and $V_{e}$ are the solid/liquid interfacial area of the dendrite and the envelope volume, respectively. The second identity is simply to express $K_{d}$ in terms of the solid/liquid interfacial area concentration, $S_{s}$, an easily measured stereological quantity. One thus obtains

$$
\beta_{d}=\frac{3 \sqrt{5} S_{s}}{\phi_{e} S_{e}\left(1-\varepsilon_{s i}\right)^{3 / 2}}
$$

\section{E. Evaluation of the Extradendritic Permeability}

The evaluation of $\beta_{l}$ needs a few more steps. It is clear from Eq. [12] that $\beta_{l}$ has to correctly represent two limiting cases: $\varepsilon_{l} \rightarrow 1$ (a single dendrite) and $\beta_{d} \rightarrow \infty$ (packed bed of solid spheres). In the former case, de Groh et al.$^{[6]}$ have proposed a correlation for the settling ratio, namely,

$$
C_{\varepsilon}^{s}=\frac{1}{C_{p}\left(\phi_{e}\right)} \frac{2 \beta_{d}^{2}\left[1-\tanh \left(\beta_{d}\right) / \beta_{d}\right]}{2 \beta_{d}^{2}+3\left[1-\tanh \left(\beta_{d}\right) / \beta_{d}\right]}
$$

where $\phi_{e}$ is the sphericity of the dendrite envelope. The correction factor, $C_{p}\left(\phi_{e}\right)$, accounts for the nonspherical shape of the dendrite envelope, while the second term on the right-hand side of Eq. [20] describes the effect of the permeability of the dendrite envelope and is based on the analytical results of Neale et al. ${ }^{[16]}$

In the other limiting case (i.e., packed bed of solid spheres), the well-known free-surface cell model attributed to Happel ${ }^{[19]}$ gives

$$
\begin{aligned}
C_{\varepsilon}^{m} & =\frac{2+\frac{4}{3} \eta^{5}}{2-3 \eta+3 \eta^{5}-2 \eta^{6}}, \quad \text { with } \\
\eta & =\left(1-\varepsilon_{l}\right)^{1 / 3}
\end{aligned}
$$

An expression for $C_{\varepsilon}$, which encompasses both limiting cases, can simply be taken as the product $C_{\varepsilon}^{s} C_{\varepsilon}^{m}$. Converting to the parameter, $\beta_{l}$, using Eqs. [7] and [11], one thus obtains

$$
\begin{aligned}
\beta_{l}= & \frac{9}{2}\left(1-\varepsilon_{l}\right) \frac{2+\frac{4}{3} \eta^{5}}{2-3 \eta+3 \eta^{5}-2 \eta^{6}} \\
& \left.\cdot \frac{1}{C_{p}\left(\phi_{e}\right)} \frac{2 \beta_{d}^{2}\left[1-\tanh \left(\beta_{d}\right) / \beta_{d}\right]}{2 \beta_{d}^{2}+3\left[1-\tanh \left(\beta_{d}\right) / \beta_{d}\right]}\right\}^{1 / 2}
\end{aligned}
$$

The function $C_{p}\left(\phi_{e}\right)$ which accounts for the nonspherical envelope shape has been experimentally determined in the packed bed regime for liquid fractions up to $0.7 .^{[20]}$ It is widely accepted that the Kozeny-Carman equation is also valid for nonspherical particles provided that the interfacial area concentration is used as the characteristic length. This suggests that

$$
C_{p}\left(\phi_{e}\right)=\phi_{e}^{2} \text { for } 0.7>\varepsilon_{l}>0.0
$$

The shape function, however, has not been evaluated in the free particle regime, with the only empirical correlation available in the limit of a single equiaxed dendrite as proposed by de Groh et al. ${ }^{[6]}$ In the absence of reliable information on $C_{p}\left(\phi_{e}\right)$ within the range of $1>\varepsilon_{l}>0.7$, the correlation of de Groh et al. ${ }^{[6]}$ is generalized to dilute systems in this work:

$$
C_{p}\left(\phi_{e}\right)=1.26 \log _{10}\left(\frac{\phi_{e}}{0.163}\right) \text { for } 1>\varepsilon_{l}>0.7
$$

\section{F. Evaluation of the Index $n$}

The last parameter, $n$, can be determined by comparing the present model consisting of Eqs. [12] and [22] with the analytical results obtained by Neale et al.$^{[16]}$ for a swarm of permeable spheres for different values of $\beta_{d}$. The best approximation is achieved when

$$
n=0.176 \log _{10} \beta_{d}+0.275
$$

as can be seen from Figure 2. It should be noted that although the results of Neale et al., ${ }^{[16]}$ which are obtained from the cell theory first proposed by Happel, ${ }^{[19]}$ are only approximate, several more recent studies ${ }^{[26,27]}$ have indicated that these closed-form results are remarkably accurate.

\section{G. Model Summary}

The present model to calculate the solid/liquid interfacial drag in equiaxed solidification is summarized in Table I. The parameters $\varepsilon_{s}, \varepsilon_{s i}, S_{s}, S_{e}$, and $\phi_{e}$ are the necessary inputs. Theoretically, the volume fractions (e.g., $\varepsilon_{s}$ and $\varepsilon_{s i}$ ) are primary variables to be calculated from transport equations, and $S_{s}, S_{e}$, and $\phi_{e}$ can be modeled as functions of certain geometric parameters and the volume fractions in question. ${ }^{[8]}$ In particular, it is shown in Reference [8] that (1) the solid/interdendritic liquid interfacial area concentration, $S_{s}$, is inversely proportional to a representative interdendritic arm spacing, 


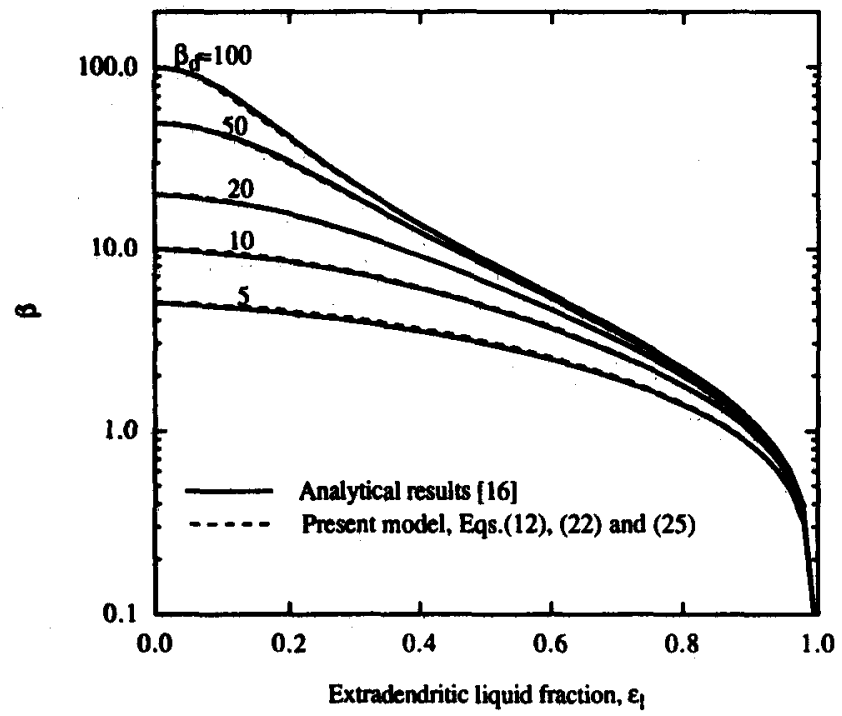

Fig. 2-Comparison of the present correlation (Eq. [13]) with analytical results of Neale $e t$ al..$^{\text {16l }}$ for different values of $\beta_{d}$, the dimensionless inverse permeability of the dendrite envelope.

Table I. Summary of the Drag Model

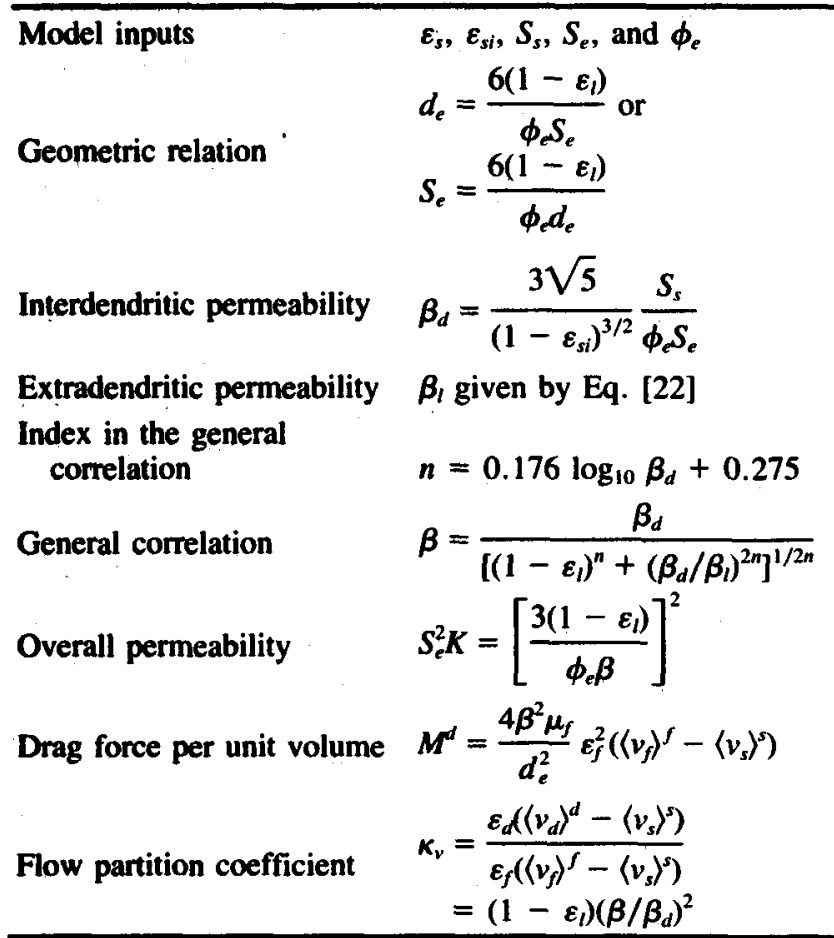

which, in turn, evolves according to the $1 / 3$ power of the local solidification time due to coarsening of the dendrite arms, and (2) the envelope area concentration, $S_{e}$, is proportional to the $1 / 3$ power of the local equiaxed grain density, which, in turn, evolves according to nucleation kinetics and the advective transport of free equiaxed crystals. Both of these structural parameters, as well as the internal solid fraction $\varepsilon_{s i}$, are routinely used in advanced solidification models. ${ }^{[8,21]}$

Experimentally, the parameters $\varepsilon_{s}$ and $S_{s}$ can be measured using quenched specimens. ${ }^{[5,22]}$ The envelope area concentration $S_{e}$ may be determined like $S_{s}$, after the dendrite envelope is manually drawn by connecting the primary and secondary arm tips in a micrograph of a quenched microstructure. The internal solid fraction $\varepsilon_{s i}$ can then be estimated from the same micrograph. Alternatively, a simple calculation method for $\varepsilon_{s i}$ has been proposed by Jang and Hellawell ${ }^{[23]}$ and de Groh et al.$^{[6]}$ that is based on estimates of a few geometrical quantities associated with the dendrite morphology. The last parameter, the envelope sphericity $\phi_{e}$, can be evaluated from Eq. [5] after $S_{e}$ and $\varepsilon_{l}$ are determined as described previously and the dendrite envelope diameter $d_{e}$ is calculated from the relation $\pi / 6 d_{e}^{3}=\left(1-\varepsilon_{l}\right) V_{0}$. The dendrite envelope sphericity $\phi_{e}$ and diameter $d_{e}$ may also be estimated by assuming a simple shape for the dendrite envelope, as illustrated by Ahuja ${ }^{[2]}$ and de Groh et al. ${ }^{[6]}$ It should be pointed out that the envelope sphericity effect is of secondary importance in determining the multiparticle interfacial drag and, thus, a crude estimate will suffice.

\section{RESULTS AND DISCUSSION}

The present model encompasses many important limiting cases, which include the single equiaxed dendrite ${ }^{[6]}$ and packed beds of impermeable ${ }^{[19]}$ and permeable spheres. ${ }^{[16]}$ In the following, comparisons are made with other available theoretical results and experimental data for both globular and dendritic grains. Since the present correlation identically reduces to the one by de Groh et $a .^{[6]}$ in the single equiaxed dendrite limit, the following discussion concentrates on multiparticle systems. For convenience of discussion, the packed bed regime is assumed to be defined by the validity range of the Kozeny-Carman equation; i.e., $0<\varepsilon_{t}<0.7$, as found in Figure 3(b). Hence, the free particle regime covers $0.7<\varepsilon_{l}<1$. Note that the preceding criterion is based on the extradendritic liquid fraction, as the particles may be porous. Physically, the transition from the packed bed to the free particle regimes can be expected to occur over a range of $\varepsilon_{l}$ (between 0.5 and 0.7 ), depending on the shape of the crystals and the packing arrangement.

\section{A. Globular Grains}

For globular grains, the envelope coincides with the solid-liquid interface, so that $S_{e}=S_{s}$ and $\varepsilon_{l}=\varepsilon_{f}$. Since $\varepsilon_{d}=0$ and $\beta_{d} \rightarrow \infty$, the general correlation (Eq. [12]), reduces to

$$
\beta=\left\{\frac{9}{2}\left(1-\varepsilon_{l}\right) \frac{2+4 / 3 \eta^{5}}{2-3 \eta+3 \eta^{5}-2 \eta^{6}} \frac{1}{C_{p}\left(\phi_{e}\right)}\right\}^{1 / 2}
$$

Combining Eqs. [11], [23], [24], and [26], it is interesting to note that the relationship between $S_{e}^{2} K$ and $\varepsilon_{l}$ is universal for all sphericities in the packed bed regime (i.e., $0<\varepsilon_{l}<0.7$ ), whereas the relation in the free particle regime (i.e., $0.7<\varepsilon_{l}<1$ ) does depend on the sphericity. Nonetheless, the area concentration $S_{e}$ is a useful lengthscale to nondimensionalize the permeability $K$. Figure 3(a) shows a comparison of $S_{e}^{2} K$ as calculated from Eqs. [11] and [26] and various experimental data. The portion of the theoretical curve in the free particle 


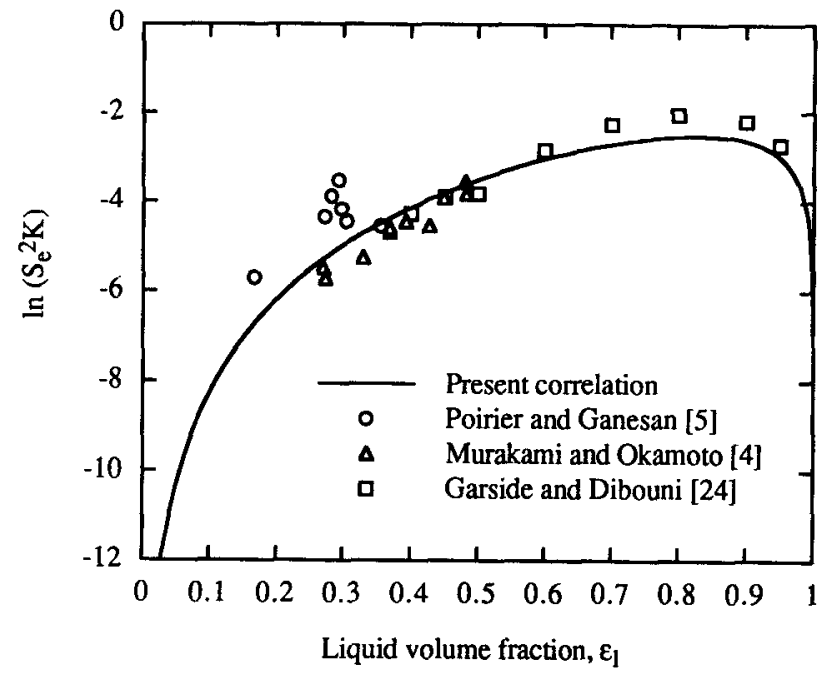

(a)

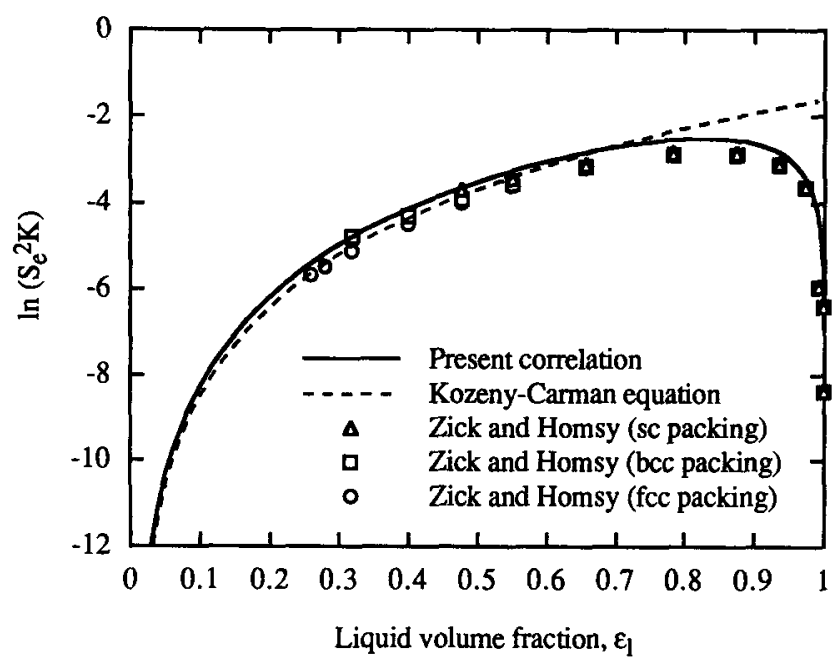

(b)

Fig. 3-Comparisons of the present model for globular dendrites $\left(\phi_{e}=1\right)$ with $(a)$ experimental data of Garside and Dibouni, ${ }^{1241}$ Murakami and Okamoto, ${ }^{[4]}$ and Poirier and Ganesan, ${ }^{[5]}$ and (b) Kozeny-Carman equation and the numerical solutions of Zick and Homsy. ${ }^{\mid 251}$

regime is only drawn for $\phi_{e}=1$ (i.e., spherical grains). of the experimental data included in Figure 3(a), Murakami and Okamoto's ${ }^{[4]}$ and Poirier and Ganesan's ${ }^{[5]}$ data are especially relevant since they were obtained for alloy systems. Only the experimental data for globular grains from Poirier and Ganesan ${ }^{[5]}$ have been included in this figure. These two sets of data cover only the packed bed regime, where the product $S_{e}^{2} K$ is independent of the sphericity of the globular grains. To further verify the model prediction in the free particle regime for spherical grains, we resort to Garside and Dibouni's data. ${ }^{24]}$ These authors have summarized results of a wide variety of sedimentation and fluidization experiments reported in the literature for solid/liquid systems. The most reliable values of the settling ratio at high liquid fractions were provided in Table II of their article. These values are also plotted in Figure 3(a) to best represent the data range of all experiments reported in the literature for perfect spheres. It can be seen from Figure 3 that the present correlation gives reasonable agreement with all experimental data over the full range of liquid fractions.

Several analytical or semianalytical relations for calculating the permeability in a mushy zone consisting of globular crystals have been proposed in the solidification literature. One is the well-known Kozeny-Carman equation, which, for globular grains, can be written as

$$
S_{e}^{2} K=\varepsilon_{l}^{3} / 5
$$

This empirical correlation is generally considered to be valid only in the packed bed regime. Another theoretical result, recommended by Poirier and Ganesan, ${ }^{[5]}$ is a numerical solution obtained by Zick and Homsy ${ }^{[25]}$ for dilute particulate systems of solid spheres. It is of interest to compare the present model prediction represented by Eq. [26] with these two results. As can be seen in Figure 3(b), the present model prediction agrees fairly well with the more exact solution of Zick and Homsy ${ }^{[25]}$ in the free particle regime, where the Kozeny-Carman equation leads to significant overpredictions. In the packed bed regime $\left(\varepsilon_{l}<0.7\right)$, the present prediction is in excellent agreement with both the Kozeny-Carman equation and Zick and Homsy's solutions. ${ }^{[25]}$ This lends support to the present correlation as a generalized drag formula for globular grains and all liquid fractions. As shown in Section B, Zick and Homsy's result ${ }^{[25]}$ cannot be used for dendritic grains and all liquid fractions, because it accounts for only a single interfacial length scale, namely, the sphere diameter.

\section{B. Dendritic Grains}

For systems composed of multiple dendritic grains, only limited experimental data were reported for the packed bed regime by Piwonka and Flemings ${ }^{[3]}$ and Poirier and Ganesan. ${ }^{[5]}$ In the absence of extradendritic liquid $\left(\varepsilon_{l}=0\right)$, these packed bed data essentially confirm the validity of the Kozeny-Carman equation (Eq. [18]), for the interdendritic permeability. ${ }^{[5] *}$ Recall that the

\footnotetext{
*The most recent packed bed data of Poirier and co-workers ${ }^{[28.29]}$ for equiaxed dendritic structures also feature no extradendritic liquid and are shown in Refs. 28 and 29 to be well correlated by Eq. [18] with $\varepsilon_{l}=0$.
}

present model reduces to Eq. [18] for $\varepsilon_{l}=0$. However, several of Poirier and Ganesan's data correspond to a "dendritic-globular" structure, where the micrographs allow the identification of a significant extradendritic liquid fraction, in addition to the interdendritic liquid. Thus, together with the comparisons shown in de Groh et $a l .^{[6]}$ for the single equiaxed dendrite $\left(\varepsilon_{l}=1\right)$, these selected data should provide some limited validation of the full interfacial drag model shown in Table I.

Since no values of $\varepsilon_{d}, S_{e}$, and $\phi_{e}$ were reported, we must seek a comparison with the dendritic-globular data in Poirier and Ganesan ${ }^{[5]}$ under certain assumptions. First, in the packed bed regime, where the experimental data lie, a sensitivity analysis of the sphericity effect indicates that the predicted values of $S_{e}^{2} K$ differ by only 3 pct for $\phi_{e}$ between 0.75 and unity. Therefore, $\phi_{e}=1$ can be safely used in the comparison between theory and 
experiment. Second, the envelope surface area, $A_{e}$, is assumed to be equal to the solid/liquid interfacial area, $A_{s}$, under the particular experimental conditions of Poirier and Ganesan. The interfacial area concentration, $S_{e}$, is then equal to $S_{s}$, with the latter quantity provided in Poirier and Ganesan. ${ }^{[5]}$ The assumption can be expected to be reasonable because the data of Poirier and Ganesan $^{[5]}$ correspond to slightly dendritic crystals. For example, at the same liquid fraction $\left(\varepsilon_{f}=0.294\right), S_{e}$ for Case 4(a) in Table 1 of their article, which corresponds to the globular structure, is identical to $S_{s}$ for Case 4(b) corresponding to the dendritic-globular structure. The same conclusion can be reached for Cases $8(a)$ and $8(b)$ in the same table. Based on these assumptions, Figure 4 displays the model predictions for a globular system $\left(\varepsilon_{s i}=1\right)$ and a dendritic system with $\varepsilon_{s i}=0.92$, in addition to the experimental data of Poirier and Ganesan ${ }^{[5]}$ for dendritic-globular grains. It is worth noting that the dendritic system has a lower permeability than the globular system at the same total liquid fraction (or solid fraction). It is also apparent that without accounting for the porosity of the grains (i.e., $\varepsilon_{s i}=1$ ), the permeability would be significantly overpredicted. It can further be seen that the curve with $\varepsilon_{s i}=0.92$ matches reasonably well with the data. From the micrographs shown in Poirier and Ganesan, ${ }^{[5]}$ this value of the internal solid fraction appears to be quite reasonable. More quantitative validation, however, must await future experiments in which all parameters $\varepsilon_{s}, \varepsilon_{s i}, S_{s}, S_{e}$, and $\phi_{e}$ are measured. Recall that the $\varepsilon_{l}=1$ limit in Figure 4 corresponds to a single equiaxed dendrite, for which full experimental validation of the present correlation has been provided in de Groh et al.$^{\left[{ }^{[6]}\right.}$ but cannot be shown in Figure 4 because the ordinate scale is inappropriate for this limiting case.

In order to examine the effects of the extradendritic liquid fraction in more detail, a parametric study was performed for $S_{s} / S_{e}=0.1, \phi_{e}=1$, and various internal solid fractions. Figures 5(a) and (b) show the overall permeability, nondimensionalized with $S_{e}^{2}$, and the flow partition coefficient, $\kappa_{v}$, respectively, as a function of the

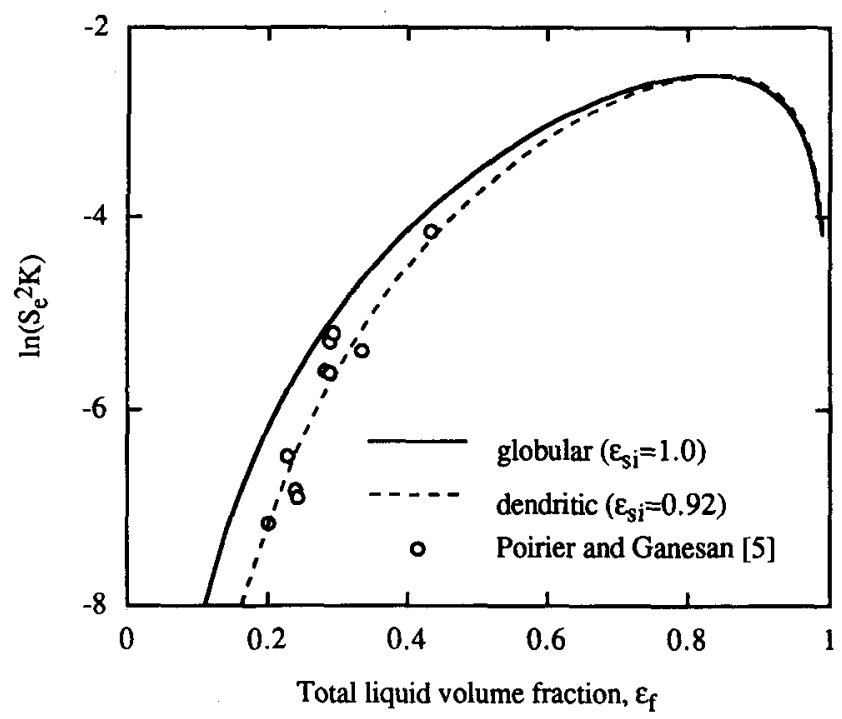

Fig. 4-Comparison of the present model with experimental data of Poirier and Ganesan ${ }^{|5|}$ for dendritic grains. extradendritic liquid fraction, $\varepsilon_{l}$. The curves with $\varepsilon_{s i}=$ 0.01 correspond to the $\mathrm{NH}_{4} \mathrm{Cl}-\mathrm{H}_{2} \mathrm{O}$ analogue alloy, for which very low internal solid fractions have been reported. ${ }^{[6,23]}$ As expected, it can be seen that systems consisting of more porous dendrites have higher permeabilities at the same extradendritic liquid fraction, particularly in the densely packed regime (Figure 5(a)). In the free particle regime, the permeability or drag is rather insensitive to the porosity of the dendrites, because the flow around the dendrites is predominant. This is also evident from Figure 5(b), which shows that the portion of the flow through the dendrites approaches zero in the free particle regime (i.e., larger $\left.\varepsilon_{l}\right)$. Furthermore, it is also consistent with the single crystal experiments of References 2 and 6, which showed no appreciable change in the interfacial drag as secondary dendrite arms were added to the internal dendritic structure of the models. On the other hand, in the packed bed regime, the flow partition coefficient quickly increases as $\varepsilon_{l}$ decreases and reaches unity at $\varepsilon_{l}=0$, at which point all flow must be through the interdendritic spaces. The effect of the porosity of the dendrites on the flow partition coefficient is also shown in Figure 5(b). In particular, note that the interdendritic liquid is already nearly immobilized when $\varepsilon_{l}>0.5$, even if a dendrite is highly porous $\left(e . g ., \varepsilon_{s i} \approx 0.01\right.$ for the $\mathrm{NH}_{4} \mathrm{Cl}-\mathrm{H}_{2} \mathrm{O}$ crystal). This is due to the fact that the complex solid/liquid interfacial structure exhibits a large resistance to fluid flow. Finally, Figure 5 also shows why Zick and Homsy's result for solid spheres ${ }^{[25]}$ cannot be used for equiaxed dendritic grains and all liquid fractions: all curves corresponding to different $\varepsilon_{s i}$ would collapse into a single one, and the highly variable nature of the flow partitioning between the extra- and interdendritic liquids would not be taken into account.

\section{CONCLUSIONS}

Through use of the porous dendrite envelope concept and based on the multiphase approach, a generalized model for the multiparticle interfacial drag in equiaxed solidification has been developed for the full range of solid fractions. The final drag or permeability correlation reflects the effects of numerous structural parameters, including the internal porosity of dendritic grains, the interdendritic arm spacings, the sphericity of the dendrite envelope or of globular grains, and the equiaxed grain density. Thus, the model is equally applicable to globular and dendritic morphologies. The model also provides information about the flow partitioning between the inter- and extradendritic liquids. It is demonstrated that the predictions agree well with previous experimental, analytical, empirical, and numerical results. Additional experimental validation is required for multiple equiaxed dendritic grains in the presence of significant extradendritic liquid fraction. The developed correlations for the drag and the flow partition coefficients have been incorporated into a recently proposed multiphase alloy solidification model that includes melt convection and solid transport. ${ }^{[18]}$ 


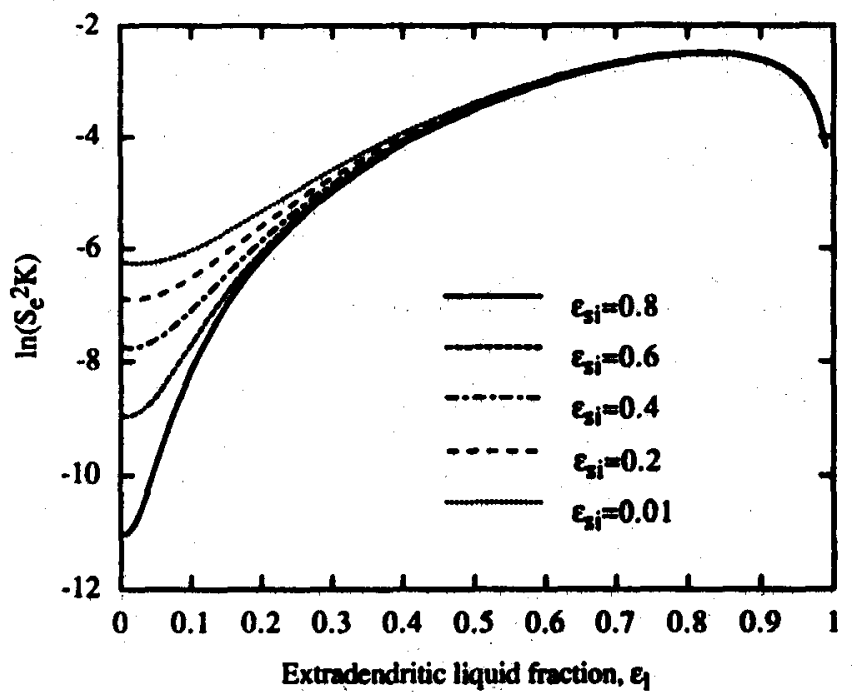

(a)

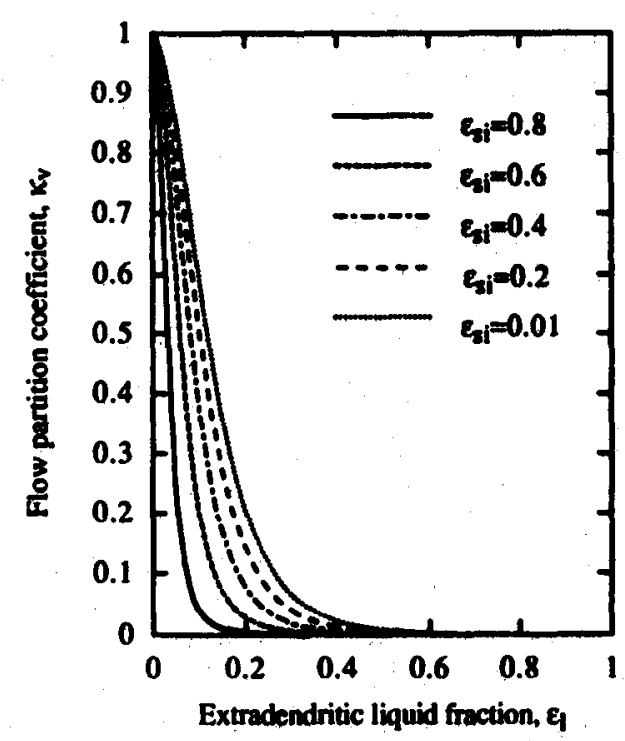

(b)

Fig. 5-(a) Permeability and (b) flow partition coefficient as functions of the extradendritic liquid fraction and internal solid fraction for $\phi_{e}=1$ and $S_{s} / S_{e}=0.1$.

\section{NOMENCLATURE}

$A$ interfacial area, $\mathrm{m}^{2}$

$C_{\mathrm{E}} \quad$ settling ratio

$C_{p} \quad$ shape factor function

$d$ diameter, $\mathrm{m}$

$K \quad$ permeability, $\mathrm{m}^{2}$

$M^{d} \quad$ solid/liquid interfacial drag per unit volume, $\mathbf{N} / \mathbf{m}^{3}$

$n \quad$ index in Eq. [10]

$S$ interfacial area concentration, $A / V_{0}, \mathrm{~m}^{-1}$

$V_{0} \quad$ volume of the control element, $\mathrm{m}^{3}$

$\left\langle v_{k}\right\rangle^{k} \quad$ volume-averaged intrinsic velocity of phase $k, \mathrm{~m} / \mathrm{s}$

Greek Symbols

$\beta \quad$ dimensionless diameter, Eq. [11]

$\varepsilon_{k} \quad$ volume fraction of phase $k$

$\varepsilon_{s i} \quad$ internal solid fraction, $\varepsilon_{s} /\left(\varepsilon_{s}+\varepsilon_{d}\right)$ $\kappa_{v} \quad$ flow partition coefficient for the interdendritic region, Eq. [13]

density, $\mathrm{kg} / \mathrm{m}^{3}$

viscosity, $\mathrm{N} \cdot \mathrm{s} / \mathrm{m}^{2}$

shape factor or sphericity of the dendrite envelope

\begin{tabular}{ll}
\multicolumn{2}{l}{ Subscripts } \\
\hline$d$ & interdendritic liquid \\
$e$ & dendrite envelope \\
$\boldsymbol{k}$ & a phase, $k=s, d$, or $l$ \\
$l$ & extradendritic liquid \\
$e$ & dendrite envelope \\
$f$ & total liquid $(d+l)$ \\
$s$ & solid
\end{tabular}

Superscripts

$\begin{array}{ll}m & \text { multiple particles } \\ s & \text { single particle }\end{array}$

\section{ACKNOWLEDGMENTS}

This work was supported by the National Science Foundation under Grant No. CTS-8957149 and the National Aeronautics and Space Administration under Grant No. NCC3-290.

\section{REFERENCES}

I. J. Ni and C. Beckermann: Metall. Trans. B, 1991, vol. 22B, pp. 349-6!.

2. S. Ahuja: MSME Thesis, The University of Lowa, lowa City, IA, 1992.

3. T.S. Piwonka and M.C. Flemings: Trans. AIME, 1966, vol. 236, pp. 1157-65.

4. K. Murakami and T. Okamoto: Acta Metall., 1984, vol. 32, Pp. $1741-44$.

S. D.R. Poirier and S. Ganesan: Mater. Sci. Eng. A, 1992, vol. 157, pp. 113-23.

6. H.C. de Groh III, P.D. Weidman, R. Zakhem, S. Ahuja, and C. Beckermann: Metall. Trans. B, 1993, vol. 24B, pp. 749-53.

7. R. Zakhem, P.D. Weidman, and H.C. de Groh III: NASA TM 105916, also in Metall. Trans. A, 1992, vol. 23A, pp. $2169-81$.

8. C.Y. Wang and C. Beckermann: Metall. Trans. A, 1993 , vol. $24 A$, pp. $2787-802$.

9. C.Y. Wang and C. Beckermann: Mater. Sci. Eng. A, 1993, vol. A171, pp. 199-211.

10. C.Y. Wang and C. Beckermann: Metall. Mater. Trans. A, 1994, vol. 25A, pp. 1081-93.

11. P.C. Carman: Flow of Gases through Porous Media, Butterworth Scientific, London, 1956.

12. M.C. Flemings: Solidification Processing, McOraw-Hill, New York, NY, 1974, p. 234.

13. J. Happel and H. Brenner: Law Reymolds Number Hydrodynamics, Noordhoff Intemational Publishing, Leyden. The Netherlands, 1973, pp. 358-422.

14. P.K. Agarwal and B.K. O'Neill: Chem. Eng. Sci., 1988, vol. 43 , pp. $2487-99$.

15. S.W. Churchill and R. Usagi: $A / C h E$ J., 1972, vol. 18, pp. 1121-28.

16. G. Neale, N. Epstein, and W. Nader: Chem. Eng. Sci., 1973. vol. 28, pp. 1865-74.

17. P.M. Adler. J. Colloid Interface Sci., 1981, vol. 81, pp. 531-35.

18. C.Y. Wang: Ph.D. Thesis, The University of lowa, lowa City, IA, 1994.

19. J. Happel: AIChE J., 1958, vol. 13, pp. 122-25.

20. I.F. Macdonald, M.S. El-Sayed, K. Mow, and F.A. Dullien: Ind. Eng. Chem. Fundam., 1977, vol. 18, pp. 199-208. 
21. M. Rappaz and D.M. Stefanescu: Metals Handbook, 9th ed., ASM, Metals Park, OH, 1988, vol. 15, pp. 883-91.

22. S.P. Marsh and M.E. Glicksman: in Modeling of Casting, Welding and Advanced Solidification Processes VI, T.S. Piwonka, V. Voller, and L. Kategerman, eds., TMS, Warrendale, PA, 1993, pp. 55-62.

23. J. Jang and A. Hellawell: Ironmaking and Steelmaking, 1991, vol. 18, pp. 275-83.

24. J. Garside and M.R. Al-Dibouni: Ind. Eng. Chem. Process Des. Dev., 1977, vol. 16, pp. 206-14.
25. A.A. Zick and G.M. Homsy: J. Fluid Mech., 1982, vol. 115, pp. 13-26.

26. R.H. Davis and H.A. Stone: Chem. Eng. Sci., 1993, vol. 48, pp. 3993-4005.

27. G. Mo and A.S. Sangani: Phys. Fluids, 1994, vol. 6, pp. 1637-52.

28. D.R. Poirier and P. Ocansey: Mater. Sci. Eng. A, 1993, vol. A171, pp. 231-40.

29. P. Ocansey, M.S. Bhat, D.R. Poirier, and T.L. Finn: in Light Metals 1994, TMS, Warrendale, PA, 1994, pp. 807-12. 